IJMMS 26:12 (2001) 753-758

PII. S0161171201006068

http://ijmms.hindawi.com

(C) Hindawi Publishing Corp.

\title{
ON AZUMAYA ALGEBRAS WITH A FINITE AUTOMORPHISM GROUP
}

\author{
GEORGE SZETO and LIANYONG XUE
}

(Received 26 October 2000)

\begin{abstract}
Let $B$ be a ring with $1, C$ the center of $B$, and $G$ a finite automorphism group of $B$. It is shown that if $B$ is an Azumaya algebra such that $B=\oplus \sum_{g \in G} J_{g}$ where $J_{g}=$ $\{b \in B \mid b x=g(x) b$ for all $x \in B\}$, then there exist orthogonal central idempotents $\left\{f_{i} \in C \mid i=1,2, \ldots, m\right.$ for some integer $\left.m\right\}$ and subgroups $H_{i}$ of $G$ such that $B=$ $\left(\oplus \sum_{i=1}^{m} B f_{i}\right) \oplus D$ where $B f_{i}$ is a central Galois algebra with Galois group $\left.H_{i}\right|_{B f_{i}} \cong H_{i}$ for each $i=1,2, \ldots, m$ and $D$ is contained in $C$.
\end{abstract}

2000 Mathematics Subject Classification. 16S35, 16W20.

1. Introduction. Let $A$ be an Azumaya algebra, $G$ a finite algebra automorphism group of $A$, and $J_{g}=\{a \in A \mid a x=g(x) a$ for all $x \in A\}$ for each $g \in G$. In [6], it was shown that $J_{g} J_{h}=J_{g h}$ for all $g, h \in G$. In [2], let $B$ be a separable algebra over a commutative ring $R$ and $G$ a finite algebra automorphism group of $B$. Assume that $B=\oplus \sum_{g \in G} J_{g}$ where $J_{g}$ are similarly defined as for $A$. Then, $B$ is a central Galois algebra with Galois group $G$ if and only if for each $g \in G, J_{g} J_{g^{-1}}=C$, the center of $B$. Thus, any Azumaya algebra $B$ with a finite algebra automorphism group $G$ such that $B=\oplus \sum_{g \in G} J_{g}$ is a central Galois algebra with Galois group $G$. By changing the algebra automorphism group $G$ to a ring automorphism group $G$, the purpose of the present paper is to generalize the above fact. We will show that if $B$ is an Azumaya $C$-algebra with a finite ring automorphism group $G$ such that $B=\oplus \sum_{g \in G} J_{g}$, then there exist orthogonal central idempotents $\left\{f_{i} \in C \mid i=1,2, \ldots, m\right.$ for some integer $\left.m\right\}$ and subgroups $H_{i}$ of $G$ such that $B=\left(\oplus \sum_{i=1}^{m} B f_{i}\right) \oplus B f$ where $B f_{i}$ is a central Galois algebra with Galois group $\left.H_{i}\right|_{B f_{i}} \cong H_{i}$ for each $i=1,2, \ldots, m, f=1-\sum_{i=1}^{m} f_{i}$, and $B f=C f$. Since a Galois algebra $B$ with Galois group $G$ is an Azumaya algebra such that $B=\oplus \sum_{g \in G} J_{g}$, our result can be applied to Galois algebras. Moreover, if $B$ is a separable extension of $B^{G}$ such that $B=\oplus \sum_{g \in G} J_{g}$, then the direct summand $B f$ is a commutative Galois algebra with Galois group $\left.G\right|_{B f} \cong G$. An example is given to demonstrate the results and to illustrate that an Azumaya algebra $B$ such that $B=\oplus \sum_{g \in G} J_{g}$ is not necessarily a Galois algebra with Galois group $G$.

2. Definitions and notations. Throughout, $B$ will represent a ring with $1, C$ the center of $B, G$ a ring automorphism group of $B$ of order $n$ for some integer $n$, and $B^{G}$ the set of elements in $B$ fixed under each element in $G$. We denote $J_{g}=\{b \in B \mid b x=$ $g(x) b$ for all $x \in B\}$ and $I_{g}=B J_{g} \cap C$ for each $g \in G$.

Let $A$ be a subring of a ring $B$ with the same identity 1 . We denote $V_{B}(A)$ the commutator subring of $A$ in $B$. We follow the definitions of a Galois extension, a separable 
extension, and an Azumaya algebra as given in $[1,5,7]$. The ring $B$ is called a separable extension of $A$ if there exist $\left\{a_{i}, b_{i}\right.$ in $B, i=1,2, \ldots, m$ for some integer $\left.m\right\}$ such that $\sum a_{i} b_{i}=1$, and $\sum b a_{i} \otimes b_{i}=\sum a_{i} \otimes b_{i} b$ for all $b$ in $B$ where $\otimes$ is over $A$. An Azumaya algebra is a separable extension of its center. The ring $B$ is called a Galois extension of $B^{G}$ with Galois group $G$ if there exist elements $\left\{a_{i}, b_{i}\right.$ in $\left.B, i=1,2, \ldots, m\right\}$ for some integer $m$ such that $\sum_{i=1}^{m} a_{i} g\left(b_{i}\right)=\delta_{1, g}$ for each $g \in G$. The algebra $B$ is called a Galois algebra over $R$ if $B$ is a Galois extension of $R$ which is contained in $C$, and $B$ is called a central Galois extension if $B$ is a Galois extension of $C$.

3. The structure theorem. In this section, we assume that $B$ is an Azumaya $C$ algebra with a finite ring automorphism group $G$ such that $B=\oplus \sum_{g \in G} J_{g}$. We will show a structure theorem for such a $B$. We begin with some properties of the $C$-module $J_{g}$ for $g \in G$ similar to those as for a Galois algebra (see [4, Proposition 2]).

LEMMA 3.1. For all $g, h \in G$,

(1) $J_{g} J_{h}=I_{g} J_{g h}=I_{h} J_{g h}$ where $I_{g}=B J_{g} \cap C$ and $I_{h}=B J_{h} \cap C$.

(2) There is a unique idempotent $e_{g} \in C$ such that $B J_{g}=B e_{g}$ and $J_{g} J_{g^{-1}}=e_{g} C$.

Proof. (1) Since $B$ is an Azumaya $C$-algebra and $B J_{g}$ is an ideal of $B, B J_{g}=B I_{g}$ (see [1, Proposition 1.11, page 46]). By hypothesis, $B=\oplus \sum_{g \in G} J_{g}$, so $B J_{h}=\sum_{g \in G} J_{g} J_{h}$. Noting that $J_{g} J_{h} \subset J_{g h}$ and $B=\oplus \sum_{g \in G} J_{g h}$, we have that $B J_{h}=\oplus \sum_{g \in G} J_{g} J_{h}$. Hence $\oplus \sum_{g \in G} J_{g} J_{h}=B J_{h}=B I_{h}=\oplus \sum_{g \in G} J_{g h} I_{h}$. Thus, $J_{g} J_{h}=I_{h} J_{g h}$. Similarly, $J_{g} J_{h}=I_{g} J_{g h}$.

(2) By (1), $J_{g} J_{h}=I_{g} J_{g h}$ for all $g, h \in G$. By letting $h=1$, we have $I_{g} J_{g}=J_{g} J_{1}=$ $J_{g} C=J_{g}$, and by letting $h=g^{-1}$, we have $J_{g} J_{g^{-1}}=I_{g} J_{1}=I_{g} C=I_{g}$. Thus, $\left(I_{g}\right)^{2}=$ $I_{\mathfrak{g}} J_{\mathfrak{g}} J_{\mathfrak{g}^{-1}}=J_{\mathfrak{g}} J_{g^{-1}}=I_{\mathfrak{g}}$. Moreover, since $B=\oplus \sum_{g} \in G J_{\mathfrak{g}}$ is an Azumaya $C$-algebra, $J_{\mathfrak{g}}$ is a finitely generated and projective $C$-module for each $g \in G$. Hence $B J_{g} \cong B \otimes_{C} J_{g}$ is a finitely generated and projective ideal of $B$. This implies that $I_{g}\left(=B J_{g} \cap C\right)$ is a finitely generated and projective ideal of $C$. But $\left(I_{g}\right)^{2}=I_{g}$, so $I_{g}=C e_{g}$ for some idempotent $e_{g} \in C$ (see [4, Lemma 2] and [3, Theorem 76]). Therefore, $B J_{g}=B I_{g}=B e_{g}$ and $J_{g} J_{g^{-1}}=I_{g}=e_{g} C$. Since $e_{g}$ is the identity of $B e_{g}$, it is unique.

By Lemma 3.1(2), for each $g \in G$, there is a unique idempotent $e_{g} \in C$ such that $B J_{g}=B e_{g}$. The Boolean algebra generated by the elements $\left\{e_{g} \mid g \in G\right.$ and $\left.B J_{g}=B e_{g}\right\}$ is denoted by $E$.

LEMMA 3.2. Let e be a nonzero element in $E$ of the form $e=\prod_{h \in H} e_{h}$ for some maximum subset $H$ of $G$. Then $H$ is a subgroup of $G$ and $h(e)=e$ for each $h \in H$.

Proof. For any $g, h \in H$,

$$
B e_{g} e_{h}=\left(B J_{g}\right)\left(B J_{h}\right)=B\left(J_{g} J_{h}\right)=B\left(I_{g} J_{g h}\right)=\left(B I_{g}\right)\left(B J_{g h}\right)=B e_{g} e_{g h} .
$$

Hence $e_{g} e_{h}=e_{g} e_{g h}$. Thus, $e_{g} e_{h}=e_{g} e_{h}^{2}=e_{g} e_{g h} e_{h}$. Therefore, $e=e e_{g h}$. Thus, $g h \in H$ by the maximality of $H$. Since $G$ is finite, that $g h \in H$ whenever $g, h \in H$ implies that $H$ is a subgroup of $G$. Noting that, for a subgroup $H, g_{H} g^{-1}=H$ for all $g \in H$, we have that

$$
g(B e)=g\left(B\left(\Pi_{h \in H} J_{h}\right)\right)=B\left(\Pi_{h \in H} \mathcal{g}\left(J_{h}\right)\right)=B\left(\Pi_{h \in H} J_{g h g^{-1}}\right)=B\left(\Pi_{h \in H} J_{h}\right)=B e
$$

for each $g \in H$. Hence, $g(e)=e$ for each $g \in H$ because $e$ is the identity of $B e$. 
Next we show that $\left.H\right|_{B e}$ is an algebra automorphism group.

LEMMA 3.3. Let e be a nonzero element in $E$ of the form $e=\Pi_{h \in H} e_{h}$ for some maximum subset $H$ of $G$. Then $h$ restricted to $C e$ is an identity for each $h \in H$.

Proof. For any $h \in H$ and $b \in J_{h}, b c=h(c) b$ for all $c \in C$, so $(c-h(c)) b=0$. Hence $(c-h(c)) J_{h}=\{0\}$. Therefore $B(c-h(c)) e_{h}=(c-h(c)) B e_{h}=(c-h(c)) B J_{h}=$ $B(c-h(c)) J_{h}=\{0\}$. Thus, $(c-h(c)) e_{h}=0$. But $e=\Pi_{h \in H} e_{h}$, so $(c-h(c)) e=0$. Moreover, $h(e)=e$ for each $h \in H$ by Lemma 3.2, so $0=(c-h(c)) e=(c-h(c)) h(e)=$ $\operatorname{ch}(e)-h(c) h(e)=c e-h(c e)$, that is, $h(c e)=c e$ for all $c \in C$.

LEMMA 3.4. Let $J_{h}^{(B f)}=\{b \in B f \mid b x=h(x) b$ for all $x \in B f\}$ for any $f \in E$ and $h \in G$. If $h(f)=f$, then $J_{h}^{(B f)}=f J_{h}$.

PRoof. It is clear that $f J_{h} \subset J_{h}^{(B f)}$. Conversely, for any $b \in J_{h}^{(B f)}, b=f b$ and $b x=$ $h(x) b$ for each $x \in B f$. Hence for any $y \in B, b y=(f b) y=b(y f)=h(y f) b=$ $h(y) f b=h(y) b$. Therefore, $b \in J_{h}$, and so $b=f b \in f J_{h}$. Thus, $J_{h}^{(B f)}=f J_{h}$.

Let $e$ and $H$ be given as in Lemma 3.2. We have a structure theorem for the Azumaya $C e$-algebra $B e$ with an algebra automorphism group $\left.H\right|_{B e} \cong H$ and for the Azumaya $C$-algebra $B$ with a ring automorphism group $G$, respectively.

THEOREM 3.5. Lete be a nonzero element in $E$ of the form $e=\prod_{h \in H} e_{h}$ for some maximum subset $H$ of $G$. Then Be is a central Galois algebra with Galois group $\left.H\right|_{B e} \cong H$.

Proof. By Lemma 3.2, $H$ is a subgroup of $G$ and $h(e)=e$ for any $h \in H$. By Lemma 3.3, $h$ restricted to $C e$ is an identity for each $h \in H$. Hence $\left.H\right|_{B e}$ is a $C e$-algebra automorphism group of $B e$. Since $B$ is an Azumaya $C$-algebra, $B e$ is an Azumaya $C e$-algebra (see [1, Proposition 1.11, page 46]). By Lemma 3.4, $J_{h}^{(B e)}=e J_{h}$ for each $h \in H$, so $B e=\oplus \sum_{g \in G} J_{g} e=\oplus \sum_{g \in H} e J_{g} \oplus \sum_{g \notin H} e J_{g}$. Since $H$ is a maximum subset of $G$ such that $e=\prod_{h \in H} e_{h}, e e_{g}=0$ for each $g \notin H$. This implies that $B e J_{g}=B e e_{g}=$ $\{0\}$. Therefore, $e J_{g}=\{0\}$ for each $g \notin H$. Thus, $B e=\oplus \sum_{g \in H} e J_{g}=\oplus \sum_{g \in H} J_{g}^{(B e)}$. Moreover, $J_{h}^{(B e)} J_{h^{-1}}^{(B e)}=\left(e J_{h}\right)\left(e J_{h^{-1}}\right)=e J_{h} J_{h^{-1}}=e e_{h} C=C e$ which is the center of $B e$ by Lemma 3.1. Thus, $B e$ is a central Galois algebra over $C e$ with Galois group $\left.H\right|_{B e}$ (see [2, Theorem 1]). Next, we claim that $\left.H\right|_{B e} \cong H$. Since $e \neq 0,\{0\} \neq B e=B e e_{h}=$ $B e J_{h}=B J_{h}^{(B e)}$ for each $h \in H$. Hence $J_{h}^{(B e)} \neq\{0\}$ for each $h \in H$. Now, if $\left.h\right|_{B e}=1$, then $\{0\} \neq C e=J_{h}^{(B e)}=e J_{h} \subset C \cap J_{h}=J_{1} \cap J_{h}$. But $B=\oplus \sum_{g \in G} J_{g}$, so $J_{1}=J_{h}$. Therefore $h=1$. This implies that $\left.h\right|_{B e} \neq 1$ whenever $h \neq 1$ in $H$. Thus, $\left.H\right|_{B e} \cong H$.

TheOREM 3.6. Let $B$ be an Azumaya $C$-algebra with a finite ring automorphism group $G$ such that $B=\oplus \sum_{g \in G} J_{g}$, then there exist orthogonal idempotents $\left\{f_{i} \in C \mid i=\right.$ $1,2, \ldots, m$ for some integer $m\}$ and subgroups $H_{i}$ of $G$ such that $B=\left(\oplus \sum_{i=1}^{m} B f_{i}\right) \oplus C f$ where $B f_{i}$ is a central Galois algebra with Galois group $\left.H_{i}\right|_{B f_{i}} \cong H_{i}$ for each $i=$ $1,2, \ldots, m$ and $f=1-\sum_{i=1}^{m} f_{i}$.

Proof. Let $\left\{f_{i} \in E \mid i=1,2, \ldots, k\right\}$ be the set of all distinct nonzero elements in $E$ of the form $f_{i}=\Pi_{h \in H_{i}} e_{h}$ for some maximum subset (subgroup) $H_{i}$ of $G$ as given in Lemma 3.2. Then they are orthogonal. Hence $B=\left(\oplus \sum_{i=1}^{k} B f_{i}\right) \oplus B f$ where $f=1-$ $\sum_{i=1}^{k} f_{i}$ such that $B f_{i}$ is a central Galois algebra with Galois group $\left.H_{i}\right|_{B f_{i}} \cong H_{i}$ for each 
$i=1,2, \ldots, k$ by Theorem 3.5. Next, we claim that $B f=C f$. Since $\left\{f_{i} \mid i=1,2, \ldots, k\right\}$ is the set of all distinct nonzero elements in $E$ of the form $f_{i}=\Pi_{h \in H_{i}} e_{h}$ for some maximum subset (subgroup) $H_{i}$ of $G, g$ permutes the set $\left\{f_{i} \mid i=1,2, \ldots, k\right\}$ for each $g \in G$. Hence $g(f)=f$ for each $g \in G$. Hence, by Lemma 3.4, $J_{g}^{(B f)}=f J_{g}$ for each $g \in G$. Therefore, $B f=\oplus \sum_{g \in G} J_{g} f=\oplus \sum_{g \in G} J_{g}^{(B f)}$ is an Azumaya $C f$-algebra with a finite ring automorphism group $\left.G\right|_{B f}$. If $J_{g}^{(B f)}=\{0\}$ for each $g \neq 1$ in $G$, then $B f=J_{1}^{(B f)}$ $=f J_{1}=C f$, and so we are done. If $J_{g}^{(B f)} \neq\{0\}$ for some $g \neq 1$ in $G$, we can repeat the above argument to have more direct summands of central Galois algebras. Since $E$ is finite, we have only finitely many central orthogonal idempotents $\left\{f_{i} \in E \mid i=\right.$ $1,2, \ldots, m$ for some integer $m\}$ such that $B=\left(\oplus \sum_{i=1}^{m} B f_{i}\right) \oplus B f$ where $B f_{i}$ is a central Galois algebra with Galois group $\left.H_{i}\right|_{B f_{i}} \cong H_{i}$ for each $i=1,2, \ldots, m$ and $B f=C f$. This completes the proof.

REMARK 3.7. Theorem 3.6 generalizes the following theorem of Harada (see [2, Theorem 1]):

Let $B$ be a separable $R$-algebra with automorphism group $G$. If $B=\oplus \sum_{g \in G} J_{g}$ and $J_{g} J_{g^{-1}}=C$ for each $g \in G$, then $B$ is a central Galois algebra with Galois group $G$.

REMARK 3.8. Any Galois algebra with Galois group $G$ satisfies the conditions as given in Theorem 3.6. There are Azumaya $C$-algebras $B$ such that $B=\oplus \sum_{g \in G} J_{g}$, but $B$ is not a Galois algebra with Galois group $G$ (see Example 3.11). However, for a Galois extension $B$ of $B^{G}$ with Galois group $G$, the condition that $B$ is a Galois algebra with Galois group $G$ and that $B=\oplus \sum_{g \in G} J_{g}$ are equivalent as given by the following proposition.

Proposition 3.9. For a Galois extension $B$ of $B^{G}$ with Galois group $G, B$ is a Galois algebra with Galois group $G$ if and only if $B=\oplus \sum_{g \in G} J_{g}$.

Proof. Since $B$ is a Galois extension of $B^{G}$ with Galois group $G, V_{B}\left(B^{G}\right)=\oplus \sum_{g \in G} J_{g}$ (see [4, Proposition 1]). Hence $B=\oplus \sum_{g \in G} J_{g}$ if and only if $V_{B}\left(B^{G}\right)=B$, that is, $B^{G} \subset C$.

As an application of Theorem 3.6, we obtain a structure theorem for a separable extension $B$ of $B^{G}$ such that $B=\oplus \sum_{g \in G} J_{g}$.

THEOREM 3.10. Let $B$ be a separable extension of $B^{G}$ such that $B=\oplus \sum_{g \in G} J_{g}$, then there exist orthogonal idempotents $\left\{f_{i} \in C \mid i=1,2, \ldots, m\right.$ for some integer $\left.m\right\}$ and subgroups $H_{i}$ of $G$ such that $B=\left(\oplus \sum_{i=1}^{m} B f_{i}\right) \oplus B f$ where $B f_{i}$ is a central Galois algebra with Galois group $\left.H_{i}\right|_{B f_{i}} \cong H_{i}$ for each $i=1,2, \ldots, m, f=1-\sum_{i=1}^{m} f_{i}$, and $B f=C f$ is $a$ commutative Galois algebra with Galois group $\left.G\right|_{B f} \cong G$ if $f \neq 0$.

PRoof. For any $a \in B^{G}$ and $b=\sum_{g \in G} b_{g} \in B$ where $b_{g} \in J_{g}, b_{g} a=g(a) b_{g}=a b_{g}$ for each $g \in G$, so $b a=\sum_{g \in G} b_{g} a=a \sum_{g \in G} b_{g}=a b$ for any $b \in B$. Thus, $a \in C$ for any $a \in B^{G}$. Therefore, $B^{G} \subset C$. Noting that $B$ is a separable algebra over $B^{G}$, we have that $B$ is an Azumaya $C$-algebra. But $B=\oplus \sum_{g \in G} J_{g}$, so, by Theorem 3.6, there exist orthogonal idempotents $\left\{f_{i} \in C \mid i=1,2, \ldots, m\right.$ for some integer $\left.m\right\}$ and subgroups $H_{i}$ of $G$ such that $B=\left(\oplus \sum_{i=1}^{m} B f_{i}\right) \oplus B f$ where $B f_{i}$ is a central Galois algebra with Galois group $\left.H_{i}\right|_{B f_{i}} \cong H_{i}$ for each $i=1,2, \ldots, m, B f=C f$, and $f=1-\sum_{i=1}^{m} f_{i}$. Thus, it suffices to show that $B f(=C f)$ is a commutative Galois algebra with Galois group 
$\left.G\right|_{B f} \cong G$ in case $f \neq 0$. In fact, since $B^{G}$ is contained in $C$ and $B$ is separable over $B^{G}, C$ is separable over $B^{G}$ (see [1, Theorem 3.8, page 55]), and so $C f$ is separable over $B^{G} f$ (see [1, Proposition 1.11, page 46]). Moreover, since $f \in C^{G}, B^{G} f \subset\left(B^{G} f\right)^{G} \subset(C f)^{G}$. Hence $C f$ is separable over $(C f)^{G}$ (see [1, Proposition 1.11, page 46]). Furthermore, by Lemma 3.4, $J_{g}^{(C f)}=J_{g}^{(B f)}=f J_{g}$ for each $g \in G$ so $J_{g}^{(C f)} \subset C \cap J_{g}=\{0\}$ for each $g \neq 1$ in $G$. This implies that $\left.g\right|_{C f} \neq$ identity whenever $g \neq 1$ in $G$ (for $J_{1}^{(C f)}=C f$ ). Thus, $\left.G\right|_{B f} \cong G$ and $B f(=C f)$ is a commutative Galois algebra with Galois group $\left.G\right|_{B f} \cong G$ (see [2, Proposition 2]). This completes the proof.

We conclude the present paper with an example to demonstrate the results in Theorem 3.6 and illustrate that an Azumaya $C$-algebra $B$ such that $B=\oplus \sum_{g \in G} J_{g}$, but not necessarily a Galois algebra with Galois group $G$.

EXAMPLE 3.11. Let $\mathbb{R}[i, j, k]$ be the real quaternion algebra over the field of real numbers $\mathbb{R}, \mathbb{Z}$ the integer ring, $D=(\mathbb{Z}+\sqrt{-1} \mathbb{Z}) \otimes_{\mathbb{Z}}(\mathbb{Z}+\sqrt{-1} \mathbb{Z}), B=\mathbb{R}[i, j, k] \oplus D$, and $G=$ $\left\{1, g_{i}, g_{j}, g_{k}\right\}$ where $g_{i}\left(a, d_{1} \otimes d_{2}\right)=\left(i a i^{-1}, \bar{d}_{1} \otimes d_{2}\right), g_{j}\left(a, d_{1} \otimes d_{2}\right)=\left(j a j^{-1}, d_{1} \otimes \bar{d}_{2}\right)$, and $g_{k}\left(a, d_{1} \otimes d_{2}\right)=\left(k a k^{-1}, \bar{d}_{1} \otimes \bar{d}_{2}\right)$, for all $\left(a, d_{1} \otimes d_{2}\right)$ in $B$, where $\bar{d}$ is the conjugate of the complex number $d$. Then,

(1) The center of $B$ is $C=\mathbb{R} \oplus D$.

(2) $B$ is an Azumaya $C$-algebra.

(3) $J_{1}=C=\mathbb{R} \oplus D, J_{g_{i}}=\mathbb{R}(i, 0), J_{g_{j}}=\mathbb{R}(j, 0), J_{g_{k}}=\mathbb{R}(k, 0)$. Hence $B=\oplus \sum_{g \in G} J_{g}$.

(4) By (3), $J_{g} J_{g^{-1}}=C(1,0)$ for each $g \neq 1$ in $G$. Hence, $f_{1}=(1,0)$ is the only nonzero element in $E$ of the form $f_{1}=\Pi_{h \in H_{1}} e_{h}$ for some maximum subset $H_{1}$ of $G$ (here $\left.H_{1}=G\right)$ and $f=1-f_{1}=(0,1 \otimes 1)$.

(5) $B=\left(\oplus \sum_{i=1}^{m} B f_{i}\right) \oplus C f$ where $m=1, B f_{i}$ is a central Galois algebra with Galois group $\left.H_{i}\right|_{B f_{i}} \cong H_{i}$ for each $i=1,2, \ldots, m$.

(6) $B^{G}=\mathbb{R} \oplus(\mathbb{Z} \otimes \mathbb{Z})=\mathbb{R} \oplus \mathbb{Z}$.

(7) Since $D$ is not separable over $\mathbb{Z}, B$ is not separable over $B^{G}(=\mathbb{R} \oplus \mathbb{Z})$. Hence $B$ is not a Galois algebra with Galois group $G$.

ACKNOWLEDGEMENT. This paper was written under the support of a Caterpillar Fellowship at Bradley University. We would like to thank Caterpillar Inc. for the support.

\section{REFERENCES}

[1] F. DeMeyer and E. Ingraham, Separable Algebras over Commutative Rings, Lecture Notes in Mathematics, vol. 181, Springer-Verlag, Berlin, 1971. MR 43\#6199. Zbl 215.36602.

[2] M. Harada, Supplementary results on Galois extension, Osaka J. Math. 2 (1965), 343-350. MR 33\#151. Zbl 178.36903.

[3] T. Kanzaki, On Galois algebra over a commutative ring, Osaka J. Math. 2 (1965), 309-317. MR 33\#150. Zbl 163.28802.

[4] I. Kaplansky, Commutative Rings, Allyn and Bacon, Massachusetts, 1970. MR 40\#7234. Zbl 203.34601.

[5] P. Nuss, Extensions galoisiennes non commutatives: normalité, cohomologie non abélienne [Noncommutative Galois extensions: normality, nonabelian cohomology], Comm. Algebra 28 (2000), no. 7, 3223-3251 (French). MR 2001d:16050. Zbl 01475110.

[6] A. Rosenberg and D. Zelinsky, Automorphisms of separable algebras, Pacific J. Math. 11 (1961), 1109-1117. MR 26\#6215. Zbl 116.02501. 
[7] G. Szeto and L. Xue, On characterizations of a center Galois extension, Int. J. Math. Math. Sci. 23 (2000), no. 11, 753-758. MR 2001c:16061. Zbl 01501976.

George SZeto: Department of Mathematics, Bradley UniVersity, Peoria, IL 61625, USA E-mail address: szeto@hi11top.brad1ey.edu

Lianyong Xue: Department of Mathematics, Bradley University, Peoria, IL 61625, USA E-mail address: 1xue@hi11 top.brad1ey.edu 


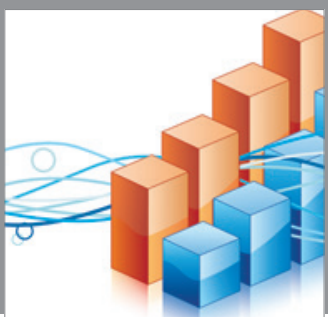

Advances in

Operations Research

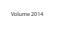

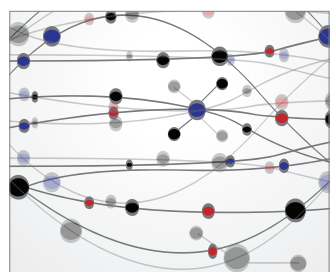

\section{The Scientific} World Journal
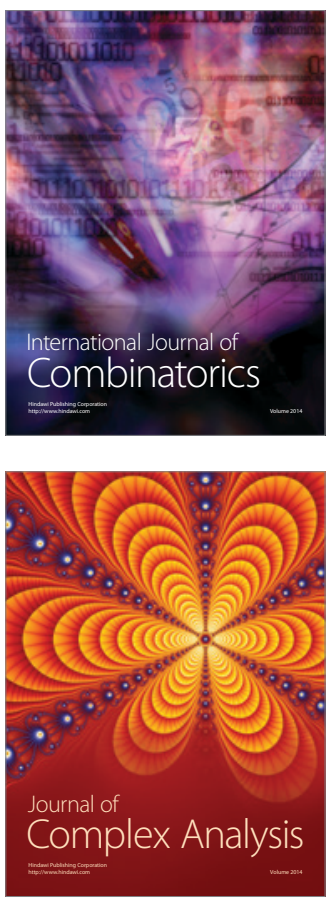

International Journal of

Mathematics and

Mathematical

Sciences
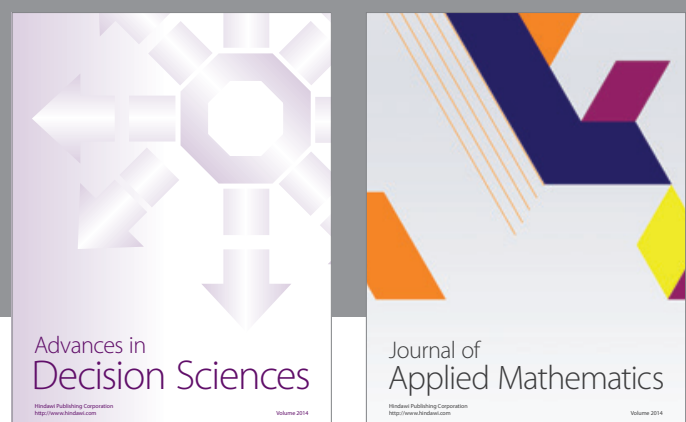

Journal of

Applied Mathematics
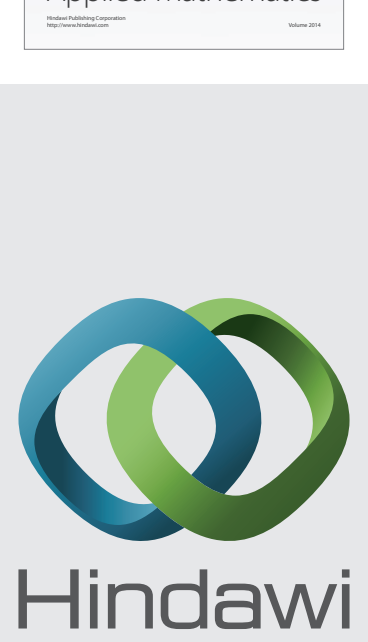

Submit your manuscripts at http://www.hindawi.com
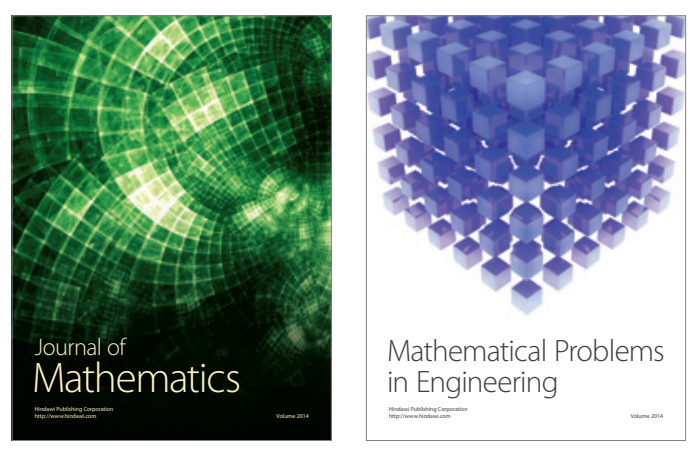

Mathematical Problems in Engineering
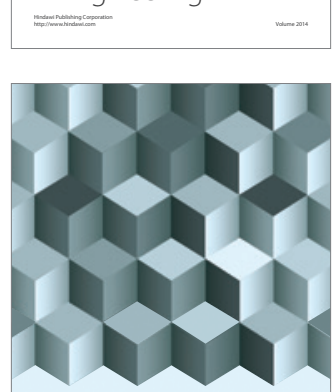

Journal of

Function Spaces
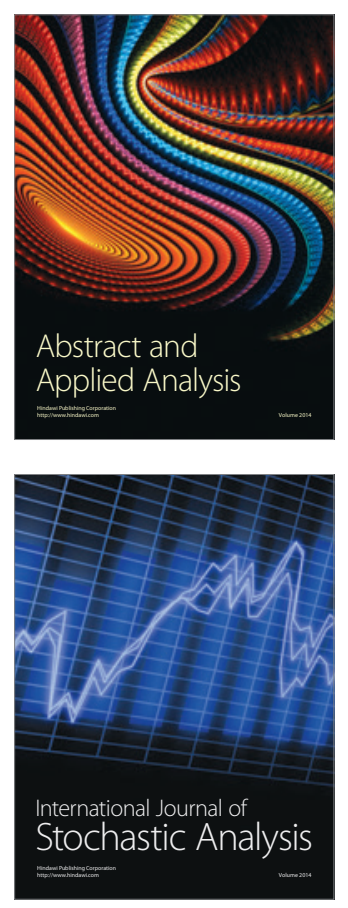

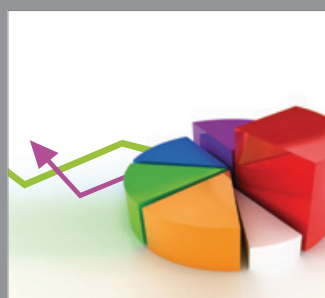

ournal of

Probability and Statistics

Promensencen
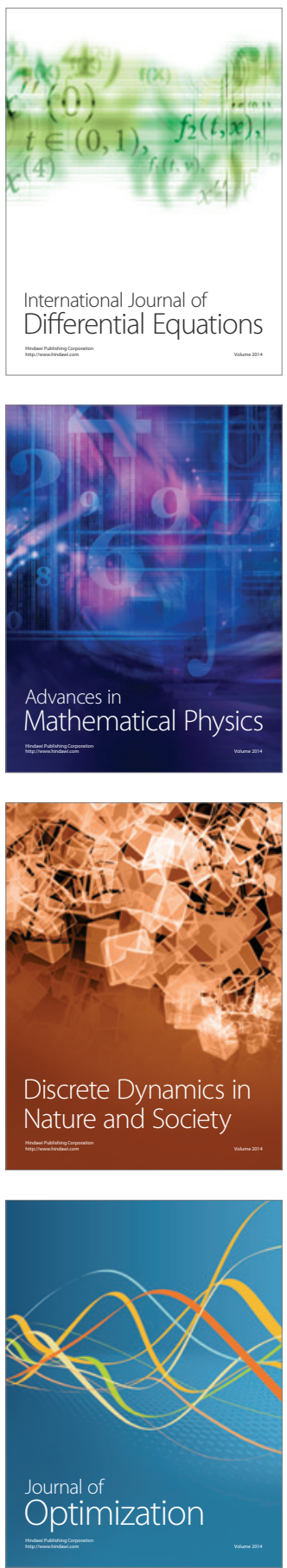